\title{
Stable weak solutions to weighted Kirchhoff equations of Lane-Emden type
}

Yunfeng Wei ${ }^{1 *} \mathbb{D}$, Hongwei Yang ${ }^{2}$ and Hongwang Yu

\section{"Correspondence:}

weiyunfeng@nau.edu.cn

'School of Statistics and

Mathematics, Nanjing Audit

University, Nanjing, 211815, P.R.

China

Full list of author information is

available at the end of the article

\begin{abstract}
This paper is concerned with the Liouville type theorem for stable weak solutions to the following weighted Kirchhoff equations:

$$
\begin{aligned}
& -M\left(\int_{\mathbb{R}^{N}} \xi(z)\left|\nabla_{G} u\right|^{2} d z\right) \operatorname{div}_{G}\left(\xi(z) \nabla_{G} u\right) \\
& =\eta(z)|u|^{p-1} u, \quad z=(x, y) \in \mathbb{R}^{N}=\mathbb{R}^{N_{1}} \times \mathbb{R}^{N_{2}},
\end{aligned}
$$

where $M(t)=a+b t^{k}, t \geq 0$, with $a, b, k \geq 0, a+b>0, k=0$ if and only if $b=0$. Let $N=N_{1}+N_{2} \geq 2, p>1+2 k$ and $\xi(z), \eta(z) \in L_{\text {loc }}^{1}\left(\mathbb{R}^{N}\right) \backslash\{0\}$ be nonnegative functions such that $\xi(z) \leq C\|z\|_{G}^{\theta}$ and $\eta(z) \geq C^{\prime}\|z\|_{G}^{d}$ for large $\|z\|_{G}$ with $d>\theta-2$. Here $\alpha \geq 0$ and $\|z\|_{G}=\left(|x|^{2(1+\alpha)}+|y|^{2}\right)^{\frac{1}{2(1+\alpha)}} \cdot \operatorname{div}_{G}\left(\right.$ resp., $\left.\nabla_{G}\right)$ is Grushin divergence (resp., Grushin gradient). Under some appropriate assumptions on $k, \theta, d$, and $N_{\alpha}=N_{1}+(1+\alpha) N_{2}$, the nonexistence of stable weak solutions to the problem is obtained. A distinguished feature of this paper is that the Kirchhoff function $M$ could be zero, which implies that the above problem is degenerate.
\end{abstract}

MSC: $35 \mathrm{~J} 60 ; 35 \mathrm{~J} 15 ; 35 \mathrm{H} 20 ; 35 \mathrm{~B} 53$

Keywords: Liouville type theorem; Stable weak solutions; Weighted Kirchhoff equations; Grushin operator; Lane-Emden nonlinearity

\section{Introduction and main result}

In this work, we are interested in the nonexistence of stable weak solutions for the weighted Kirchhoff equations

$$
\begin{array}{r}
-M\left(\int_{\mathbb{R}^{N}} \xi(z)\left|\nabla_{G} u\right|^{2} d z\right) \operatorname{div}_{G}\left(\xi(z) \nabla_{G} u\right) \\
=\eta(z)|u|^{p-1} u, \quad z \in \mathbb{R}^{N}=\mathbb{R}^{N_{1}} \times \mathbb{R}^{N_{2}},
\end{array}
$$

where $M$ is a nonnegative continuous function which will be given later. Here and thereafter, we assume that $p>1$ and $\xi(z), \eta(z) \in L_{\text {loc }}^{1}\left(\mathbb{R}^{N}\right) \backslash\{0\}$ are nonnegative functions. For $z=(x, y) \in \mathbb{R}^{N}=\mathbb{R}^{N_{1}} \times \mathbb{R}^{N_{2}}$ and $\alpha \geq 0$, we define the Grushin gradient $\nabla_{G}$ and Grushin

(c) The Author(s) 2021. This article is licensed under a Creative Commons Attribution 4.0 International License, which permits use, sharing, adaptation, distribution and reproduction in any medium or format, as long as you give appropriate credit to the original author(s) and the source, provide a link to the Creative Commons licence, and indicate if changes were made. The images or other third party material in this article are included in the article's Creative Commons licence, unless indicated otherwise in a credit line to the material. If material is not included in the article's Creative Commons licence and your intended use is not permitted by statutory regulation or exceeds the permitted use, you will need to obtain permission directly from the copyright holder. To view a copy of this licence, visit http://creativecommons.org/licenses/by/4.0/. 
divergence $\operatorname{div}_{G}$ as follows:

$$
\begin{aligned}
& \nabla_{G} u=\left(\nabla_{x} u,(1+\alpha)|x|^{\alpha} \nabla_{y} u\right), \\
& \operatorname{div}_{G} \mathbf{v}=\operatorname{div}_{x} \mathbf{v}+(1+\alpha)|x|^{\alpha} \operatorname{div}_{y} \mathbf{v} .
\end{aligned}
$$

The Grushin operator $\Delta_{G}$ is denoted by

$$
\Delta_{G} u=\operatorname{div}_{G}\left(\nabla_{G} u\right)=\Delta_{x} u+(1+\alpha)^{2}|x|^{2 \alpha} \Delta_{y} u,
$$

which is just the well-known Laplace operator when $\alpha=0$.

The anisotropic dilation attached to $\Delta_{G}$ is defined by

$$
\tau_{\delta}(z)=\left(\delta x, \delta^{1+\alpha} y\right), \quad \delta>0, z=(x, y) \in \mathbb{R}^{N_{1}} \times \mathbb{R}^{N_{2}} .
$$

It is easy to check that

$$
d \tau_{\delta}(z)=\delta^{N_{\alpha}} d x d y=\delta^{N_{\alpha}} d z
$$

where $N_{\alpha}=N_{1}+(1+\alpha) N_{2}$ is a homogeneous dimension with respect to the dilation $\tau_{\delta}$, and $d x d y$ denotes the Lebesgue measure on $\mathbb{R}^{N_{1}} \times \mathbb{R}^{N_{2}}$. Finally, the norm of $z$ (also known as the Grushin distance) is defined by

$$
\|z\|_{G}=\left(|x|^{2(1+\alpha)}+|y|^{2}\right)^{\frac{1}{2(1+\alpha)}}, \quad z=(x, y) \in \mathbb{R}^{N}=\mathbb{R}^{N_{1}} \times \mathbb{R}^{N_{2}} .
$$

In the case $\alpha=0, \xi(z) \equiv 1$ and $M(t)=a+b t, t \geq 0$ with $a, b>0$, (1.1) becomes the following nonlocal Kirchhoff type problem:

$$
-\left(a+b \int_{\mathbb{R}^{N}}|\nabla u|^{2} d z\right) \Delta u=\eta(z)|u|^{p-1} u, \quad z \in \mathbb{R}^{N} .
$$

This problem is often referred to as being nonlocal because of the presence of the integral over the entire domain $\mathbb{R}^{N}$. This phenomenon provokes many mathematical difficulties, which makes the research on the problem particularly interesting and necessary. Besides, problem (1.2) is related to the stationary analogue of the Kirchhoff equation on a bounded domain $\Omega \subset \mathbb{R}^{N}$

$$
u_{t t}-\left(a+b \int_{\Omega}|\nabla u|^{2} d z\right) \Delta u=f(z, u)
$$

proposed by Kirchhoff in 1883 (see [20]) as generalization of the classical D'Alembert's wave equation for free vibration of elastic strings. It is important to note that equation (1.3) received great attention only after Lions [26] introduced a functional analysis approach. For recent interesting results concerning the various solutions of Kirchhoff equations and the stationary analogue of problem (1.3), please refer to $[1,4,18,19,28,29,37]$ and the references therein.

Recently, much attention has been paid to the Kirchhoff equations in the whole space $\mathbb{R}^{N}$. Most of recent results have been concerned with the existence and multiplicity of solutions when the nonlinearity is in subcritical case and critical case. For instance, in the 
paper [35], Wu considered a class of Schrödinger Kirchhoff type equations in $\mathbb{R}^{N}$ with subcritical case, by using the symmetric mountain pass theorem, four new existence results for nontrivial solutions and a sequence of high energy solutions have been obtained. In [14], Fan studied the existence and multiplicity of positive ground state solutions for a Kirchhoff type problem involving several potentials and critical nonlinearities in $\mathbb{R}^{N}$. In addition, in this article, the concentration phenomenon of positive solutions was also considered. For more similar works, we refer to $[25,27,40]$. However, compared with a vast number of existence results for entire solutions of Kirchhoff type equations, the nonexistence one is less investigated.

In this paper, the Kirchhoff function $M$ is assumed to verify

(M) $M(t)=a+b t^{k}, t \geq 0, a, b, k \geq 0, a+b>0$, and

$$
k \begin{cases}\in(0, \infty), & \text { if } b>0 \\ 0, & \text { if } b=0\end{cases}
$$

in other words, $k=0$ if and only if $b=0$. Problem (1.2) is called non-degenerate if $a>0$ and $b \geq 0$, while it is said to be degenerate if $a=0$ and $b>0$. The degenerate case is extremely interesting and is treated in well-known papers on Kirchhoff's theory, for example, see [11]. For degenerate Kirchhoff type problems, we refer to [24, 27, 36, 39]. For nondegenerate Kirchhoff type problems, we refer to [14, 25, 28, 29, 35, 40].

Set $\alpha=0$ and $M(t) \equiv 1$, problem (1.1) becomes the weighted Lane-Emden equation

$$
-\operatorname{div}(\xi(z) \nabla u)=\eta(z)|u|^{p-1} u \quad \text { in } \mathbb{R}^{N}
$$

In recent years, much attention has been focused on studying of the nonexistence and stability of solutions to nonlinear elliptic equations like (1.4). For some physical motivation and recent developments on the topic of stable solutions, we refer to [13].

Liouville type theorems for stable solutions deal with the nonexistence of this particular type of solutions. The pioneering work in this direction is due to Farina [15], where the author established thoroughly the Liouville type theorem for stable classical solutions of problem (1.4) with $\xi(z) \equiv 1 \equiv \eta(z)$. He proved that the problem does not admit a nontrivial stable $C^{2}$ solution if and only if $1<p<p_{c}(N)$, where

$$
p_{c}(N)= \begin{cases}+\infty, & \text { if } N \leq 10, \\ \frac{(N-2)^{2}-4 N+8 \sqrt{N-1}}{(N-2)(N-10)}, & \text { if } N \geq 11 .\end{cases}
$$

Moreover, this exponent is greater than the classical critical exponent $\frac{N+2}{N-2}$ [17] when $N>2$. After that, the above results have been generalized to the weighted case in [7, 10, 34]. In [10], under the restriction that the solutions are locally bounded, the authors presented the nonexistence of nontrivial stable weak solutions of problem (1.4) with $\xi(z) \equiv 1$ and $\eta(z)=|z|^{d}$. In [34], this restriction was withdrawn.

Theorem 1.1 ([34]) Let $u$ be a stable weak solution of (1.4) with $\xi(z) \equiv 1$ and $\eta(z)=|z|^{d}$, where $d>-2$. Then $u$ is a trivial solution provided $1<p<p(N, d)$. Here

$$
p(N, d)= \begin{cases}+\infty, & \text { if } N \leq 10+4 d, \\ \frac{(N-2)(N-6-2 d)-2(2+d)^{2}+2(2+d) \sqrt{(2+d)(2 N-2+d)}}{(N-2)(N-10-4 d)}, & \text { if } N>10+4 d .\end{cases}
$$


In [7], with the help of Farina's approach, Cowan and Fazly established several Liouville type theorems for stable positive classical solutions of problem (1.4) under different assumptions on $\xi$ and $\eta$. Later, several attempts have been made to extend Farina's results to the weighted quasilinear case $-\operatorname{div}\left(\xi(z)|\nabla u|^{p-2} \nabla u\right)=\eta(z)|u|^{p-1} u$, in $\mathbb{R}^{N}$, see $[5,6,22,23]$.

We now turn to the case $\alpha>0$. Then problem (1.1) is a nonlocal problem involving Grushin operator. It is well known that the Grushin operator belongs to the wide class of subelliptic operators studied by Franchi et al. in [16](see also [3]). Taking advantage of the Kelvin transform combined with the method of moving planes, the Liouville type theorem has been established by Monticelli [31] (resp., Yu [38]) for nonnegative classical (resp., weak) solutions of the problem $-\Delta_{G} u=u^{p}$ in $\mathbb{R}^{N}$, the optimal exponent is $1<p<\frac{N_{\alpha}+2}{N_{\alpha}-2}$. Recently, Duong and Nguyen [12] studied elliptic equations involving Grushin operator and advection

$$
-\Delta_{G} u+\nabla_{G} w \cdot \nabla_{G} u=\|z\|_{G}^{s}|u|^{p-1} u, \quad \text { in } \mathbb{R}^{N}, s \geq 0 .
$$

Via Farina's approach, the authors obtained several Liouville type theorems for a class of stable sign-changing weak solutions.

Very recently, Le [21] considered the elliptic problems

$$
-\operatorname{div}_{G}\left(w_{1} \nabla_{G} u\right)=w_{2} f(u), \quad \text { in } \Omega,
$$

with homogeneous Dirichlet boundary condition. By variable technique, under suitable assumptions on $\Omega, w_{1}, w_{2}$, and $f$, nonexistence of stable weak solutions has been established. When $\Omega=\mathbb{R}^{N}$ and $f$ has power or exponential growth, the author also constructed some examples to show the sharpness of his results. For other results of Liouville type theorems related to Grushin operators or more general subelliptic operators, we refer the reader to $[2,9,30,32,33]$ and the references therein.

A natural question is whether there are analogous Liouville results for Kirchhoff type equation (1.1) with $\alpha>0$ and $\xi, \eta \not \equiv 1$. The present paper is an attempt to answer this interesting question.

Motivated by the aforementioned works, we prove the nonexistence of nontrivial stable weak solution to equation (1.1). Since solutions to elliptic equations with Hardy potentials may possess singularities, it is natural to study weak solutions of (1.1) in a suitable weighted Sobolev space. Based on this reality, we define

$$
\|\psi\|_{\xi}=\left(\int_{\mathbb{R}^{N}} \xi(z)\left|\nabla_{G} \psi\right|^{2} d z\right)^{1 / 2}
$$

for $\psi \in C_{0}^{\infty}\left(\mathbb{R}^{N}\right)$ and denote by $H^{1, \alpha}\left(\mathbb{R}^{N} ; \xi\right)$ the closure of $C_{0}^{\infty}\left(\mathbb{R}^{N}\right)$ with respect to the $\|\cdot\|_{\xi}$-norm. Note that, for $\xi \in L_{\text {loc }}^{1}\left(\mathbb{R}^{N}\right)$, we have $C_{0}^{1}\left(\mathbb{R}^{N}\right) \subset H^{1, \alpha}\left(\mathbb{R}^{N} ; \xi\right)$. Denote also by $H_{\text {loc }}^{1, \alpha}\left(\mathbb{R}^{N} ; \xi\right)$ the space of all functions $u$ such that $u \psi \in H^{1, \alpha}\left(\mathbb{R}^{N} ; \xi\right)$ for all $\psi \in C_{0}^{1}\left(\mathbb{R}^{N}\right)$.

Definition 1.2 Let $X=H^{1, \alpha}\left(\mathbb{R}^{N} ; \xi\right) \cap H_{\text {loc }}^{1, \alpha}\left(\mathbb{R}^{N} ; \xi\right)$, we say that $u \in X$ is a weak solution of (1.1) if $\eta(z)|u|^{p} \in L_{\text {loc }}^{1}\left(\mathbb{R}^{N}\right)$, and for all $\psi \in C_{0}^{1}\left(\mathbb{R}^{N}\right)$ we have

$$
A \int_{\mathbb{R}^{N}} \xi(z) \nabla_{G} u \cdot \nabla_{G} \psi d z=\int_{\mathbb{R}^{N}} \eta(z)|u|^{p-1} u \psi d z
$$

where $A=a+b\|u\|_{\xi}^{2 k}$. 
Now, we give the stability definition. First, we consider that the energy functional $I$ : $X \rightarrow \mathbb{R}$ associated with (1.1) is

$$
I(u)=\frac{a}{2}\|u\|_{\xi}^{2}+\frac{b}{2(k+1)}\|u\|_{\xi}^{2(k+1)}-\frac{1}{p+1} \int_{\mathbb{R}^{N}} \eta(z)|u|^{p+1} d z .
$$

Set $E(t)=I(u+t \psi)$ for $\psi \in C_{0}^{1}\left(\mathbb{R}^{N}\right)$. Then, by routine calculations, we show $E^{\prime \prime}(0) \geq 0$ is equivalent to

$$
\begin{aligned}
p \int_{\mathbb{R}^{N}} \eta(z)|u|^{p-1} \psi^{2} d z \leq & 2 b k\|u\|_{\xi}^{2(k-1)}\left(\int_{\mathbb{R}^{N}} \xi(z) \nabla_{G} u \cdot \nabla_{G} \psi d z\right)^{2} \\
& +A \int_{\mathbb{R}^{N}} \xi(z)\left|\nabla_{G} \psi\right|^{2} d z .
\end{aligned}
$$

A weak solution $u$ of (1.1) is called stable if (1.8) holds for all $\psi \in C_{0}^{1}\left(\mathbb{R}^{N}\right)$. Therefore, if $u$ is a stable weak solution of (1.1), by Hölder's inequality and (1.8), it follows that

$$
p \int_{\mathbb{R}^{N}} \eta(z)|u|^{p-1} \psi^{2} d z \leq B \int_{\mathbb{R}^{N}} \xi(z)\left|\nabla_{G} \psi\right|^{2} d z, \quad \forall \psi \in C_{0}^{1}\left(\mathbb{R}^{N}\right),
$$

where $B=a+b(1+2 k)\|u\|_{\xi}^{2 k}$. Remark that (1.7)-(1.9) hold for all $\psi \in H^{1, \alpha}\left(\mathbb{R}^{N} ; \xi\right)$ by density arguments.

Throughout this paper, we assume that the functions $\xi(z), \eta(z)$ satisfy the following assumption:

(H) $\xi(z), \eta(z) \in L_{\mathrm{loc}}^{1}\left(\mathbb{R}^{N}\right) \backslash\{0\}$ are nonnegative functions. In addition, there exist $d>\theta-2$, $C, C^{\prime}>0$, and $R_{0}>0$ such that

$$
\xi(z) \leq C\|z\|_{G}^{\theta}, \quad \eta(z) \geq C^{\prime}\|z\|_{G}^{d}, \quad \forall\|z\|_{G} \geq R_{0} .
$$

To facilitate the writing, we denote

$$
\begin{aligned}
& \lambda_{0}=\lambda_{0}(k, \theta, d)= \begin{cases}+\infty, & k=0, \\
2-\theta+\frac{2-\theta+d}{k}, & k>0,\end{cases} \\
& \lambda_{1}=\lambda_{1}(k, \theta, d)=2-\theta+\frac{4(2-\theta+d)}{1+2 k}, \\
& \lambda_{2}=\lambda_{2}(k, \theta, d)=2-\theta+\frac{(1+\sqrt{1+2 k})(2-\theta+d)}{2 k} \quad(k>0) .
\end{aligned}
$$

Let us now state the main result of this work.

Theorem 1.3 Let $u \in X$ be a stable weak solution of problem (1.1) with $p>1+2 k$. Assume that $(M)$ and $(H)$ hold. We further suppose that one of the following conditions occurs:

$\left(H_{1}\right) k \geq 0, N_{\alpha} \leq \min \left\{\lambda_{0}, \lambda_{1}\right\}$, and $p>1+2 k$;

$\left(H_{2}\right) 0 \leq k<\frac{1}{2}, \lambda_{1}<N_{\alpha} \leq \lambda_{0}$, and $1+2 k<p<p_{c}$;

$\left(H_{3}\right) 0<k \leq \frac{1}{2}, \lambda_{0}<N_{\alpha}<\lambda_{2}$, and $\hat{p}_{c}<p<p_{c}$;

$\left(H_{4}\right) k>\frac{1}{2}, \lambda_{0}<N_{\alpha}<\lambda_{1}$, and $p>\hat{p}_{c}$;

$\left(H_{5}\right) \frac{1}{2}<k<\frac{3}{2}, N_{\alpha}=\lambda_{1}$, and $p>\frac{4}{3-2 k}$; 
$\left(H_{6}\right) \frac{1}{2}<k<\frac{3}{2}, \lambda_{1}<N_{\alpha}<\lambda_{2}$, and $\hat{p}_{c}<p<p_{c}$, with critical exponents

$$
\begin{aligned}
\hat{p}_{c} & =\hat{p}_{c}\left(k, \theta, d, N_{\alpha}\right) \\
& =1+\frac{2(2-\theta+d)\left(N_{\alpha}-4+2 \theta-d-\sqrt{\left(N_{\alpha}+d\right)^{2}-(1+2 k)\left(N_{\alpha}-2+\theta\right)^{2}}\right)}{\left(N_{\alpha}-2+\theta\right)(1+2 k)\left(N_{\alpha}-\lambda_{1}\right)} ; \\
p_{c} & =p_{c}\left(k, \theta, d, N_{\alpha}\right) \\
& =1+\frac{2(2-\theta+d)\left(N_{\alpha}-4+2 \theta-d+\sqrt{\left(N_{\alpha}+d\right)^{2}-(1+2 k)\left(N_{\alpha}-2+\theta\right)^{2}}\right)}{\left(N_{\alpha}-2+\theta\right)(1+2 k)\left(N_{\alpha}-\lambda_{1}\right)} .
\end{aligned}
$$

Then $u \equiv 0$ in $\mathbb{R}^{N}$.

Remark 1.4 Indeed, the assumption on $p$ in Theorem 1.3 is equivalent to

$$
N_{\alpha}<2-\theta+\frac{2(2-\theta+d)(p+\sqrt{p(p-1-2 k)})}{(1+2 k)(p-1)} .
$$

Remark 1.5 If $k=0$ (i.e., $b=0$ ), then Theorem 1.3 recovers the known result for the Grushin operator in Le [21, Proposition 3]. Furthermore, if $\alpha=k=0$, then our result recovers the previous result for elliptic problems with weights in Cowan and Fazly [7, Theorem 3]. If $\alpha=k=\theta=0$, then we have

$$
p_{c}(0,0, d, N)=1+\frac{2(2+d)(N-4-d+\sqrt{(2+d)(2 N-2+d)})}{(N-2)(N-10-4 d)} .
$$

Note that the exponent $p_{c}(0,0, d, N)$ equals $p(N, d)$ of (1.6) when $N>10+4 d$ and is the critical exponent $\bar{p}(d)$ in [10]. When $\alpha=k=\theta=d=0$, we obtain

$$
p_{c}(0,0,0, N)=1+\frac{4(N-4+2 \sqrt{N-1})}{(N-2)(N-10)},
$$

which is the critical exponent $p_{c}(N)$ in [15]. Finally, when $\alpha=\theta=0$ and $k=1$, it is not difficult to verify that $\hat{p}_{c}(1,0, d, N)\left(\right.$ resp., $\left.p_{c}(1,0, d, N)\right)$ equals $q_{1}(N, d)\left(\right.$ resp., $\left.q_{2}(N, d)\right)$ in [24].

The rest of the paper is devoted to the proof of Theorem 1.3. In the following, $C$ stands for a generic positive constant which may vary even in the same line. If this constant depends on an arbitrary small number $\varepsilon$, then we denote it by $C_{\varepsilon}$.

\section{Proof of Theorem 1.3}

We first give the following proposition, which plays a crucial role in arriving at Theorem 1.3.

Proposition 2.1 Let $u \in X$ be a stable weaksolution of (1.1) with $p>1+2 k$. Then, for every $s \in(1, h(p))$, where

$$
h(t)=-1+\frac{2(t+\sqrt{t(t-1-2 k)})}{1+2 k}, \quad t>1+2 k,
$$


and for any constant $m \geq \frac{p+s}{p-1}$, there exists a constant $C>0$ depending only on $p, s, m$, and $k$ such that

$$
\begin{aligned}
& \int_{\mathbb{R}^{N}}\left(\eta(z)|u|^{p+s}+\xi(z)\left|\nabla_{G} u\right|^{2}|u|^{s-1}\right) \varphi^{2 m} d z \\
& \quad \leq C B^{\frac{1+s}{p-1}}(1+B) \int_{\mathbb{R}^{N}} \xi(z)^{\frac{p+s}{p-1}} \eta(z)^{\frac{1+s}{1-p}}\left|\nabla_{G} \varphi\right|^{\frac{2(p+s)}{p-1}} d z
\end{aligned}
$$

holds for all functions $\varphi \in C_{0}^{1}\left(\mathbb{R}^{N}\right)$ verifying $0 \leq \varphi \leq 1$ and $\nabla_{G} \varphi=0$ in a neighborhood of $\left\{z \in \mathbb{R}^{N}: \eta(z)=0\right\}$. Here, $B$ is given by (1.9).

Proof Some ideas in this proof are inspired by $[8,12,15,21]$. Since the solution $u$ is not necessary locally bounded, to overcome this difficulty, we will construct a sequence of suitable cut-off functions. Let $n$ be a positive integer, we denote

$$
\delta_{n}(t)=\left\{\begin{array}{ll}
|t|^{\frac{s-1}{2}} t, & |t| \leq n, \\
n^{\frac{s-1}{2}} t, & |t|>n,
\end{array} \quad v_{n}(t)= \begin{cases}|t|^{s-1} t, & |t| \leq n, \\
n^{s-1} t, & |t|>n .\end{cases}\right.
$$

By a direct computation, we obtain that, for any $t \in \mathbb{R}$, there exists a positive constant $C$ depending only on $s$ such that

$$
\delta_{n}^{2}(t)=t v_{n}(t), \quad \delta_{n}^{\prime}(t)^{2} \leq \frac{(s+1)^{2}}{4 s} v_{n}^{\prime}(t), \quad \delta_{n}^{2}(t)+v_{n}^{2}(t)\left(v_{n}^{\prime}(t)\right)^{-1} \leq C|t|^{s+1} .
$$

Moreover, since $u \in H_{\mathrm{loc}}^{1, \alpha}\left(\mathbb{R}^{N}, \xi\right)$, we deduce that $\delta_{n}(u), v_{n}(u) \in H_{\mathrm{loc}}^{1, \alpha}\left(\mathbb{R}^{N}, \xi\right)$ for any $n \in \mathbb{Z}^{+}$.

For any nonnegative function, $\phi \in C_{0}^{1}\left(\mathbb{R}^{N}\right)$ satisfies $0 \leq \phi \leq 1$. Setting $\psi=v_{n}(u) \phi^{2}$ as a test function in (1.7), we have

$$
\begin{aligned}
& A \int_{\mathbb{R}^{N}} \xi(z)\left|\nabla_{G} u\right|^{2} v_{n}^{\prime}(u) \phi^{2} d z+2 A \int_{\mathbb{R}^{N}} \xi(z) \phi v_{n}(u) \nabla_{G} u \cdot \nabla_{G} \phi d z \\
& \quad=\int_{\mathbb{R}^{N}} \eta(z)|u|^{p-1} u v_{n}(u) \phi^{2} d z .
\end{aligned}
$$

Applying Young's inequality, for any $\varepsilon>0$,

$$
\begin{aligned}
& A \int_{\mathbb{R}^{N}} \xi(z)\left|\nabla_{G} u\right|^{2} v_{n}^{\prime}(u) \phi^{2} d z \\
& \leq 2 A \int_{\mathbb{R}^{N}} \xi(z)\left|v_{n}(u)\right|\left|\nabla_{G} u\right|\left|\nabla_{G} \phi\right| \phi d z+\int_{\mathbb{R}^{N}} \eta(z)|u|^{p-1} u v_{n}(u) \phi^{2} d z \\
& \leq \varepsilon A \int_{\mathbb{R}^{N}}\left(\xi(z)^{1 / 2}\left|\nabla_{G} u\right| v_{n}^{\prime}(u)^{1 / 2} \phi\right)^{2} d z \\
&+C_{\varepsilon} A \int_{\mathbb{R}^{N}}\left(\xi(z)^{1 / 2}\left|v_{n}(u)\right| v_{n}^{\prime}(u)^{-1 / 2}\left|\nabla_{G} \phi\right|\right)^{2} d z \\
&+\int_{\mathbb{R}^{N}} \eta(z)|u|^{p-1} u v_{n}(u) \phi^{2} d z \\
&= \varepsilon A \int_{\mathbb{R}^{N}} \xi(z)\left|\nabla_{G} u\right|^{2} v_{n}^{\prime}(u) \phi^{2} d z+C_{\varepsilon} A \int_{\mathbb{R}^{N}} \xi(z) v_{n}^{2}(u) v_{n}^{\prime}(u)^{-1}\left|\nabla_{G} \phi\right|^{2} d z \\
&+\int_{\mathbb{R}^{N}} \eta(z)|u|^{p-1} u v_{n}(u) \phi^{2} d z,
\end{aligned}
$$


which implies

$$
\begin{aligned}
& (1-\varepsilon) A \int_{\mathbb{R}^{N}} \xi(z)\left|\nabla_{G} u\right|^{2} v_{n}^{\prime}(u) \phi^{2} d z \\
& \quad \leq C_{\varepsilon} A \int_{\mathbb{R}^{N}} \xi(z) v_{n}^{2}(u) v_{n}^{\prime}(u)^{-1}\left|\nabla_{G} \phi\right|^{2} d z+\int_{\mathbb{R}^{N}} \eta(z)|u|^{p-1} u v_{n}(u) \phi^{2} d z .
\end{aligned}
$$

On the other hand, by virtue of the stability definition, we take $\psi=\delta_{n}(u) \phi$ in (1.9) and get

$$
\begin{aligned}
& p \int_{\mathbb{R}^{N}} \eta(z)|u|^{p-1} \delta_{n}^{2}(u) \phi^{2} d z \\
& \quad \leq B \int_{\mathbb{R}^{N}} \xi(z)\left|\nabla_{G} u\right|^{2} \delta_{n}^{\prime}(u)^{2} \phi^{2} d z+2 B \int_{\mathbb{R}^{N}} \xi(z) \delta_{n}^{\prime}(u)\left|\delta_{n}(u)\right|\left|\nabla_{G} u\right|\left|\nabla_{G} \phi\right| \phi d z \\
& \quad+B \int_{\mathbb{R}^{N}} \xi(z) \delta_{n}^{2}(u)\left|\nabla_{G} \phi\right|^{2} d z .
\end{aligned}
$$

We use Young's inequality to estimate the middle term of the right-hand side of (2.5):

$$
\begin{aligned}
& 2 B \int_{\mathbb{R}^{N}} \xi(z) \delta_{n}^{\prime}(u)\left|\delta_{n}(u)\right|\left|\nabla_{G} u\right|\left|\nabla_{G} \phi\right| \phi d z \\
& \quad \leq \varepsilon B \int_{\mathbb{R}^{N}}\left(\xi(z)^{1 / 2}\left|\nabla_{G} u\right| \delta_{n}^{\prime}(u) \phi\right)^{2} d z+C_{\varepsilon} B \int_{\mathbb{R}^{N}}\left(\xi(z)^{1 / 2}\left|\delta_{n}(u)\right|\left|\nabla_{G} \phi\right|\right)^{2} d z \\
& \quad=\varepsilon B \int_{\mathbb{R}^{N}} \xi(z)\left|\nabla_{G} u\right|^{2} \delta_{n}^{\prime}(u)^{2} \phi^{2} d z+C_{\varepsilon} B \int_{\mathbb{R}^{N}} \xi(z) \delta_{n}^{2}(u)\left|\nabla_{G} \phi\right|^{2} d z .
\end{aligned}
$$

Substituting this inequality into (2.5), one has

$$
\begin{aligned}
& p \int_{\mathbb{R}^{N}} \eta(z)|u|^{p-1} \delta_{n}^{2}(u) \phi^{2} d z \\
& \quad \leq(1+\varepsilon) B \int_{\mathbb{R}^{N}} \xi(z)\left|\nabla_{G} u\right|^{2} \delta_{n}^{\prime}(u)^{2} \phi^{2} d z+C_{\varepsilon} B \int_{\mathbb{R}^{N}} \xi(z) \delta_{n}^{2}(u)\left|\nabla_{G} \phi\right|^{2} d z .
\end{aligned}
$$

With the help of (2.3), it follows from (2.4) and (2.6) that

$$
\begin{aligned}
p \int_{\mathbb{R}^{N}} \eta(z)|u|^{p-1} \delta_{n}^{2}(u) \phi^{2} d z \leq & \frac{(1+\varepsilon)(1+s)^{2} B}{4 s} \int_{\mathbb{R}^{N}} \xi(z)\left|\nabla_{G} u\right|^{2} v_{n}^{\prime}(u) \phi^{2} d z \\
& +C_{\varepsilon} B \int_{\mathbb{R}^{N}} \xi(z) \delta_{n}^{2}(u)\left|\nabla_{G} \phi\right|^{2} d z \\
\leq & \frac{(1+\varepsilon)(1+s)^{2} B}{4 s(1-\varepsilon) A} \int_{\mathbb{R}^{N}} \eta(z)|u|^{p-1} u v_{n}(u) \phi^{2} d z \\
& +C_{\varepsilon} B \int_{\mathbb{R}^{N}} \xi(z)\left(\delta_{n}^{2}(u)+v_{n}^{2}(u) v_{n}^{\prime}(u)^{-1}\right)\left|\nabla_{G} \phi\right|^{2} d z \\
\leq & \frac{(1+\varepsilon)(1+s)^{2}(1+2 k)}{4 s(1-\varepsilon)} \int_{\mathbb{R}^{N}} \eta(z)|u|^{p-1} \delta_{n}^{2}(u) \phi^{2} d z \\
& +C_{\varepsilon} B \int_{\mathbb{R}^{N}} \xi(z)|u|^{1+s}\left|\nabla_{G} \phi\right|^{2} d z,
\end{aligned}
$$


that is,

$$
q_{\varepsilon} \int_{\mathbb{R}^{N}} \eta(z)|u|^{p-1} \delta_{n}^{2}(u) \phi^{2} d z \leq C_{\varepsilon} B \int_{\mathbb{R}^{N}} \xi(z)|u|^{1+s}\left|\nabla_{G} \phi\right|^{2} d z
$$

where $q_{\varepsilon}=p-\frac{(1+\varepsilon)(1+s)^{2}(1+2 k)}{4 s(1-\varepsilon)}$. Since $\lim _{\varepsilon \rightarrow 0^{+}} q_{\varepsilon}=q_{0}=p-\frac{(1+s)^{2}(1+2 k)}{4 s}$, we have $q_{0}>0$ under assumption on $s \in(1, h(p))$. Thus, choose small $\varepsilon>0$ such that $q_{\varepsilon}>0$. Consequently,

$$
\int_{\mathbb{R}^{N}} \eta(z)|u|^{p-1} \delta_{n}^{2}(u) \phi^{2} d z \leq C B \int_{\mathbb{R}^{N}} \xi(z)|u|^{1+s}\left|\nabla_{G} \phi\right|^{2} d z
$$

where positive constant $C$ depends only on $p, k$, and $s$.

From (2.8) and Fatou's lemma, we derive, as $n \rightarrow+\infty$,

$$
\int_{\mathbb{R}^{N}} \eta(z)|u|^{p+s} \phi^{2} d z \leq C B \int_{\mathbb{R}^{N}} \xi(z)|u|^{1+s}\left|\nabla_{G} \phi\right|^{2} d z .
$$

On the other hand, choosing $\varepsilon=1 / 2$ in (2.4) and combining (2.3) with (2.8), we can find

$$
\begin{aligned}
& A \int_{\mathbb{R}^{N}} \xi(z)\left|\nabla_{G} u\right|^{2} v_{n}^{\prime}(u) \phi^{2} d z \\
& \quad \leq C A \int_{\mathbb{R}^{N}} \xi(z) v_{n}^{2}(u) v_{n}^{\prime}(u)^{-1}\left|\nabla_{G} \phi\right|^{2} d z+2 \int_{\mathbb{R}^{N}} \eta(z)|u|^{p-1} u v_{n}(u) \phi^{2} d z \\
& \quad \leq C A \int_{\mathbb{R}^{N}} \xi(z)|u|^{1+s}\left|\nabla_{G} \phi\right|^{2} d z+2 \int_{\mathbb{R}^{N}} \eta(z)|u|^{p-1} \delta_{n}^{2}(u) \phi^{2} d z \\
& \quad \leq C(A+B) \int_{\mathbb{R}^{N}} \xi(z)|u|^{1+s}\left|\nabla_{G} \phi\right|^{2} d z .
\end{aligned}
$$

Letting $n \rightarrow+\infty$ in the above inequality, we have from Fatou's lemma that

$$
A \int_{\mathbb{R}^{N}} \xi(z)\left|\nabla_{G} u\right|^{2}|u|^{s-1} \phi^{2} d z \leq C(A+B) \int_{\mathbb{R}^{N}} \xi(z)|u|^{1+s}\left|\nabla_{G} \phi\right|^{2} d z
$$

Consequently,

$$
\int_{\mathbb{R}^{N}} \xi(z)\left|\nabla_{G} u\right|^{2}|u|^{s-1} \phi^{2} d z \leq C \int_{\mathbb{R}^{N}} \xi(z)|u|^{1+s}\left|\nabla_{G} \phi\right|^{2} d z .
$$

Now, we assert that (2.2) holds true. In fact, we can select some positive constant $m \gg 1$ such that

$$
\frac{(m-1)(p+s)}{1+s} \geq m, \quad \text { or } \quad m \geq \frac{p+s}{p-1}
$$

Recalling $0 \leq \phi(z) \leq 1$ in $\mathbb{R}^{N}$, we obtain

$$
(\phi(z))^{\frac{2(m-1)(p+s)}{1+s}} \leq(\phi(z))^{2 m}, \quad \forall z \in \mathbb{R}^{N} .
$$


Then, by (2.9) with $\phi=\varphi^{m}$ and Hölder's inequality, one sees

$$
\begin{aligned}
& \int_{\mathbb{R}^{N}} \eta(z)|u|^{p+s} \varphi^{2 m} d z \\
& \leq C B \int_{\mathbb{R}^{N}} \xi(z)|u|^{1+s} \varphi^{2(m-1)}\left|\nabla_{G} \varphi\right|^{2} d z \\
& \leq C B\left(\int_{\mathbb{R}^{N}}\left(\eta(z)^{\frac{1+s}{p+s}}|u|^{1+s} \varphi^{2(m-1)}\right)^{\frac{p+s}{1+s}} d z\right)^{\frac{1+s}{p+s}} \\
& \times\left(\int_{\mathbb{R}^{N}}\left(\xi(z) \eta(z)^{-\frac{1+s}{p+s}}\left|\nabla_{G} \varphi\right|^{2}\right)^{\frac{p+s}{p-1}} d z\right)^{\frac{p-1}{p+s}} \\
&= C B\left(\int_{\mathbb{R}^{N}} \eta(z)|u|^{p+s} \varphi^{\frac{2(m-1)(p+s)}{1+s}} d z\right)^{\frac{1+s}{p+s}} \\
& \times\left(\int_{\mathbb{R}^{N}} \xi(z)^{\frac{p+s}{p-1}} \eta(z)^{\frac{1+s}{1-p}}\left|\nabla_{G} \varphi\right|^{\frac{2(p+s)}{p-1}} d z\right)^{\frac{p-1}{p+s}} \\
& \leq C B\left(\int_{\mathbb{R}^{N}} \eta(z)|u|^{p+s} \varphi^{2 m} d z\right)^{\frac{1+s}{p+s}}\left(\int_{\mathbb{R}^{N}} \xi(z)^{\frac{p+s}{p-1}} \eta(z)^{\frac{1+s}{1-p}}\left|\nabla_{G} \varphi\right|^{\frac{2(p+s)}{p-1}} d z\right)^{\frac{p-1}{p+s}} .
\end{aligned}
$$

Hence,

$$
\int_{\mathbb{R}^{N}} \eta(z)|u|^{p+s} \varphi^{2 m} d z \leq C B^{\frac{p+s}{p-1}} \int_{\mathbb{R}^{N}} \xi(z)^{\frac{p+s}{p-1}} \eta(z)^{\frac{1+s}{1-p}}\left|\nabla_{G} \varphi\right|^{\frac{2(p+s)}{p-1}} d z .
$$

Analogously, take $\phi=\varphi^{m}$ in (2.10) and combining (2.11) with (2.12), one can achieve

$$
\begin{aligned}
\int_{\mathbb{R}^{N}} & \xi(z)\left|\nabla_{G} u\right|^{2}|u|^{s-1} \varphi^{2 m} d z \\
\leq & C \int_{\mathbb{R}^{N}} \xi(z)|u|^{1+s} \varphi^{2(m-1)}\left|\nabla_{G} \varphi\right|^{2} d z \\
\leq C B^{\frac{1+s}{p-1}} & \int_{\mathbb{R}^{N}} \xi(z)^{\frac{p+s}{p-1}} \eta(z)^{\frac{1+s}{1-p}}\left|\nabla_{G} \varphi\right|^{\frac{2(p+s)}{p-1}} d z .
\end{aligned}
$$

Therefore, combining this with (2.12), (2.2) is obtained immediately. This completes the proof.

Let $R>0, \Omega_{2 R}=B_{1}(0,2 R) \times B_{2}\left(0,2 R^{1+\alpha}\right)$, where $B_{i} \subset \mathbb{R}^{N_{i}}$, with $i=1,2$, are open balls centered at 0 , the radii are $2 R$ and $2 R^{1+\alpha}$, respectively. We consider a cut-off function $\kappa(t) \in$ $C_{0}^{\infty}([0,+\infty) ;[0,1])$ satisfying

$$
\kappa(t)= \begin{cases}1, & 0 \leq t \leq 1 \\ 0, & t \geq 2\end{cases}
$$

Moreover, we define

$$
\varphi_{1, R}(x)=\kappa\left(\frac{|x|}{R}\right), \quad x \in \mathbb{R}^{N_{1}}, \quad \varphi_{2, R}(y)=\kappa\left(\frac{|y|}{R^{1+\alpha}}\right), \quad y \in \mathbb{R}^{N_{2}}
$$


and

$$
\varphi_{R}(x, y)=\varphi_{1, R}(x) \varphi_{2, R}(y), \quad(x, y) \in \mathbb{R}^{N}=\mathbb{R}^{N_{1}} \times \mathbb{R}^{N_{2}} .
$$

The direct calculations yield

$$
\begin{aligned}
& \left|\nabla_{x} \varphi_{1, R}\right| \leq C R^{-1}, \quad\left|\nabla_{y} \varphi_{2, R}\right| \leq C R^{-(1+\alpha)}, \\
& \left|\Delta_{x} \varphi_{1, R}\right| \leq C R^{-2}, \quad\left|\Delta_{y} \varphi_{2, R}\right| \leq C R^{-2(1+\alpha)}, \\
& \left|\nabla_{G} \varphi_{R}\right|^{2}+\left|\Delta_{G} \varphi_{R}\right| \leq C R^{-2}, \quad \forall x \in \mathbb{R}^{N_{1}}, y \in \mathbb{R}^{N_{2}}, \\
& R \leq\|z\|_{G} \leq C R, \quad \forall z=(x, y) \in \Omega_{2 R} \backslash \Omega_{R},
\end{aligned}
$$

where positive constant $C$ is independent of $R$.

Proof of Theorem 1.3 By contradiction, we assume that (1.1) admits a nontrivial stable weak solution $u$. Applying (2.2) for a test function $\varphi_{R}(x, y)$, which is given by (2.13), we derive that, for all $R \geq R_{0}$, there exists a constant $C>0$ independent of $R$ such that

$$
\begin{aligned}
& \int_{\Omega_{R}}\left(\eta(z)|u|^{p+s}+\xi(z)\left|\nabla_{G} u\right|^{2}|u|^{s-1}\right) d z \\
& \quad \leq C B^{\frac{1+s}{p-1}}(1+B) R^{-\frac{2(p+s)}{p-1}} \int_{\Omega_{2 R} \backslash \Omega_{R}}\|z\|_{G}^{\frac{(p+s) \theta-(1+s) d}{p-1}} d z \\
& \quad \leq C B^{\frac{1+s}{p-1}}(1+B) R^{\mu}
\end{aligned}
$$

with

$$
\mu=N_{\alpha}-\frac{(2-\theta)(p+s)+(1+s) d}{p-1} .
$$

Here, we have utilized assumption $(H)$ and (2.14).

Evidently, if $\mu<0$ for some certain $s \in(1, h(p))$, it implies from (2.15) that

$$
\int_{\mathbb{R}^{N}}\left(\eta(z)|u|^{p+s}+\xi(z)\left|\nabla_{G} u\right|^{2}|u|^{s-1}\right) d z=0
$$

as $R \rightarrow+\infty$, i.e., $u \equiv 0$ in $\mathbb{R}^{N}$, which contradicts the assumption of $u$. Therefore, we obtain the desired conclusion.

Now, we consider the cases in which $\mu<0$. Set

$$
g(t)=\frac{(2-\theta)(t+h(t))+(1+h(t)) d}{t-1}, \quad t>1+2 k,
$$

where $h(t)$ is given by (2.1). Elementary calculations lead to

$$
\lim _{t \rightarrow(1+2 k)^{+}} h(t)=1, \quad \lim _{t \rightarrow+\infty} h(t)=+\infty, \quad h^{\prime}(t)>0, \quad t>1+2 k
$$


and

$$
\begin{aligned}
& \lim _{t \rightarrow(1+2 k)^{+}} g(t)=\lambda_{0}, \quad \lim _{t \rightarrow+\infty} g(t)=\lambda_{1}, \\
& g^{\prime}(t)=\frac{(2-\theta+d)(-2 \sqrt{t(t-1-2 k)}+t(2 k-1)+1+2 k)}{(1+2 k)(t-1)^{2} \sqrt{t(t-1-2 k)}}, \quad t>1+2 k,
\end{aligned}
$$

where $\lambda_{0}, \lambda_{1}$ are given by (1.10). By routine computations we show that if $k=0$, then $g(t)$ is strictly decreasing on $(1,+\infty)$. If $0<k<\frac{3}{2}$, then $g(t)$ is strictly increasing on $(1+$ $\left.2 k, \frac{1+2 k+2 \sqrt{1+2 k}}{3-2 k}\right)$ and strictly decreasing on $\left(\frac{1+2 k+2 \sqrt{1+2 k}}{3-2 k},+\infty\right)$, in this case, $g(t)$ achieves its maximum at $t=\frac{1+2 k+2 \sqrt{1+2 k}}{3-2 k}$ and $g\left(\frac{1+2 k+2 \sqrt{1+2 k}}{3-2 k}\right)=\lambda_{2}$, where $\lambda_{2}$ is given by (1.10). If $k \geq \frac{3}{2}$, then $g(t)$ is strictly increasing on $(1+2 k,+\infty)$. Moreover, when $0 \leq k<\frac{1}{2}, \lambda_{1}<\lambda_{0}$, when $k=\frac{1}{2}, \lambda_{1}=\lambda_{0}$, when $k>\frac{1}{2}, \lambda_{1}>\lambda_{0}$. We also have $g\left(\frac{4}{3-2 k}\right)=\lambda_{1}(k, \theta, d)$.

When $k \geq 0$ and $N_{\alpha} \leq \min \left\{\lambda_{0}, \lambda_{1}\right\}$, we have $N_{\alpha}<g(t)$ for $t>1+2 k$. Thus, if we fix $s \in$ $(1, h(p))$ sufficiently near to $h(p)$, we see that

$$
\mu=N_{\alpha}-\frac{(2-\theta)(p+s)+(1+s) d}{p-1}<0, \quad p>1+2 k
$$

It is a contradiction by letting $R \rightarrow+\infty$ in (2.15).

When $0 \leq k<\frac{1}{2}$ and $\lambda_{1}<N_{\alpha} \leq \lambda_{0}$. By the monotonicity of $g(t)$, there exists a unique critical value $p_{c}>1+2 k$ such that $N_{\alpha}<g(t)$ for $1+2 k<t<p_{c}$. So if we choose $s \in(1, h(p))$ sufficiently near to $h(p)$, we get

$$
\mu=N_{\alpha}-\frac{(2-\theta)(p+s)+(1+s) d}{p-1}<0, \quad 1+2 k<p<p_{c}
$$

which is a contradiction by taking advantage of the previous similar argument.

Assume now $0<k \leq \frac{1}{2}$ and $\lambda_{0}<N_{\alpha}<\lambda_{2}$. Make use of the monotonicity of $g(t)$, there are critical exponents $\hat{p}_{c}$ and $p_{c}$ such that $N_{\alpha}<g(t)$ for $\hat{p}_{c}<t<p_{c}$. Similar to the above argument, we get a contradiction.

Combining $g\left(\frac{4}{3-2 k}\right)=\lambda_{1}(k, \theta, d)\left(\frac{1}{2}<k<\frac{3}{2}\right)$ with the monotonicity of $g(t)$, using the similar argument above, we can always deduce a contradiction provided that one of conditions $\left(H_{4}\right),\left(H_{5}\right)$, and $\left(H_{6}\right)$ is satisfied.

In the above statements, $\hat{p}_{c}$ and $p_{c}$ can be derived from the equation $N_{\alpha}=h(p)$, which is given by (1.11). The proof is finished.

\section{Acknowledgements}

The authors would like to express their sincere gratitude to the anonymous reviewer for their valuable comments and suggestions which improved the presentation of the paper.

\section{Funding}

This work was supported by the Natural Science Foundation of the Higher Education Institutions of Jiangsu Province (Grant No. 19KJD100002), the Natural Science Foundation of Shandong Province (Grant No. ZR2018MA017), and the China Postdoctoral Science Foundation (Grant No. 2017M610436).

\section{Availability of data and materials}

Not applicable.

\section{Competing interests}

The authors declare that they have no competing interests.

\section{Authors' contributions}

Each of the authors contributed to each part of this study equally. All authors read and approved the final manuscript. 


\section{Author details}

'School of Statistics and Mathematics, Nanjing Audit University, Nanjing, 211815, P.R. China. ${ }^{2}$ College of Mathematics and Systems Science, Shandong University of Science and Technology, Qingdao, 266590, P.R. China.

\section{Publisher's Note}

Springer Nature remains neutral with regard to jurisdictional claims in published maps and institutional affiliations.

Received: 28 August 2020 Accepted: 16 December 2020 Published online: 07 January 2021

\section{References}

1. Alves, C.O., Corrêa, F.J.S.A., Ma, T.F.: Positive solutions for a quasilinear elliptic equation of Kirchhoff type. Comput. Math. Appl. 49, 85-93 (2005)

2. Anh, C.T., Lee, J., My, B.K.: On the classification of solutions to an elliptic equation involving the Grushin operator. Complex Var. Elliptic Equ. 63, 671-688 (2018)

3. Birindelli, I., Capuzzo Dolcetta, I., Cutrì, A.: Liouville theorems for semilinear equations on the Heisenberg group. Ann. Inst. Henri Poincaré, Anal. Non Linéaire 14, 295-308 (1997)

4. Chen, C.-Y., Kuo, Y.-C., Wu, T.-F.: The Nehari manifold for a Kirchhoff type problem involving sign-changing weight functions. J. Differ. Equ. 250, 1876-1908 (2011)

5. Chen, C.S.: Liouville type theorem for stable solutions of $p$-Laplace equation in $\mathbb{R}^{N}$. Appl. Math. Lett. 68, 62-67 (2017)

6. Chen, C.S., Song, H.X., Yang, H.W.: Liouville-type theorems for stable solutions of singular quasilinear in $\mathbb{R}^{N}$. Electron. J. Differ. Equ. 2018, 81 (2018)

7. Cowan, C., Fazly, M.: On stable entire solutions of semi-linear elliptic equations with weights. Proc. Am. Math. Soc. 140, 2003-2012 (2012)

8. Damascelli, L., Farina, A., Sciunzi, B., Valdinoci, E.: Liouville results for $m$-Laplace equations of Lane-Emden-Fowler type. Ann. Inst. Henri Poincaré, Anal. Non Linéaire 26, 1099-1119 (2009)

9. D’Ambrosio, L., Lucente, S.: Nonlinear Liouville theorems for Grushin and Tricomi operators. J. Differ. Equ. 193, 511-541 (2003)

10. Dancer, E.N., Du, Y.H., Guo, Z.M.: Finite Morse index solutions of an elliptic equation with supercritical exponent. J. Differ. Equ. 250, 3281-3310 (2011)

11. D'Ancona, P., Spagnolo, S.: Global solvability for the degenerate Kirchhoff equation with real analytic data. Invent. Math. 108, 247-262 (1992)

12. Duong, A.T., Nguyen, N.T.: Liouville type theorems for elliptic equations involving Grushin operator and advection. Electron. J. Differ. Equ. 2017, 108 (2017)

13. Dupaigne, L.: Stable Solutions of Elliptic Partial Differential Equations. Chapman \& Hall/CRC Monographs and Surveys in Pure and Applied Mathematics, vol. 143. Chapman \& Hall, Boca Raton (2011)

14. Fan, H.N.: Positive solutions for a Kirchhoff-type problem involving multiple competitive potentials and critical Sobolev exponent. Nonlinear Anal. 198, 111869 (2020). https://doi.org/10.1016/j.na.2020.111869

15. Farina, A.: On the classification of solutions of the Lane-Emden equation on unbounded domain of $\mathbb{R}^{N}$. J. Math. Pures Appl. 87, 537-561 (2007)

16. Franchi, B., Gutiérrez, C.E., Wheeden, R.L.: Weighted Sobolev-Poincaré inequalities for Grushin type operators. Commun. Partial Differ. Equ. 19, 523-604 (1994)

17. Gidas, B., Spruck, J.: Global and local behavior of positive solutions of nonlinear elliptic equations. Commun. Pure Appl. Math. 34, 525-598 (1981)

18. Han, Y.Z., Li, Q.W.: Threshold results for the existence of global and blow-up solutions to Kirchhoff equations with arbitrary initial energy. Comput. Math. Appl. 75, 3283-3297 (2018)

19. Huynh, N.V., Le, P.: Instability of solutions to Kirchhoff type problems in low dimension. Ann. Pol. Math. 124, 75-91 (2020)

20. Kirchhoff, G.: Mechanik. Teubner, Leipzig (1883)

21. Le, P.: Liouville theorems for stable weak solutions of elliptic problems involving Grushin operator. Commun. Pure Appl. Anal. 19, 511-525 (2020)

22. Le, P., Ho, V.: Stable solutions to weighted quasilinear problems of Lane-Emden type. Electron. J. Differ. Equ. 2018, 71 (2018)

23. Le, P., Ho, V.: Liouville results for stable solutions of quasilinear equations with weights. Acta Math. Sci. Ser. B Engl. Ed. 39, 357-368 (2019)

24. Le, P., Huynh, N.V., Ho, V.: Classification results for Kirchhoff equations in $\mathbb{R}^{N}$. Complex Var. Elliptic Equ. 64, 1146-1157 (2019)

25. Li, Q.Q., Teng, K.M., Wang, W.B., Zhang, J.: Concentration phenomenon of solutions for a class of Kirchhoff-type equations with critical growth. J. Math. Anal. Appl. 491, 124355 (2020). https://doi.org/10.1016/j.jmaa.2020.124355

26. Lions, J.-L.: On some questions in boundary value problems of mathematical physics. In: Contemporary Developments in Continuum Mechanics and Partial Differential Equations (Proc. Internat. Sympos., Inst. Mat., Univ. Fed. Rio de Janeiro, Rio de Janeiro, 1977) NorthHolland Math. Stud., vol. 30, pp. 284-346. North-Holland, Amsterdam (1978)

27. Liu, J., Liao, J.F., Tang, C.L.: Positive solutions for Kirchhoff-type equations with critical exponent in $\mathbb{R}^{N}$. J. Math. Anal. Appl. 429, 1153-1172 (2015)

28. Liu, W.J., Li, G., Hong, L.H.: General decay and blow-up of solutions for a system of viscoelastic equations of Kirchhoff type with strong damping. J. Funct. Spaces 2014, 284809 (2014). https://doi.org/10.1155/2014/284809

29. Liu, W.J., Zhu, B.Q., Li, G., Wang, D.H.: General decay for a viscoelastic Kirchhoff equation with Balakrishnan-Taylor damping, dynamic boundary conditions and a time-varying delay term. Evol. Equ. Control Theory 6, 239-260 (2017)

30. Monti, R., Morbidelli, D.: Kelvin transform for Grushin operators and critical semilinear equations. Duke Math. J. 131 167-202 (2006) 
31. Monticelli, D.D.: Maximum principles and the method of moving planes for a class of degenerate elliptic linear operators. J. Eur. Math. Soc. 12, 611-654 (2010)

32. Rahal, B:: Liouville-type theorems with finite Morse index for semilinear $\Delta_{\lambda}$-Laplace operators. NoDEA Nonlinear Differ. Equ. Appl. 25, 21 (2018). https://doi.org/10.1007/s00030-018-0512-z

33. Rahal, B.: On stale entire solutions of sub-elliptic system involving advection terms with negative exponents and weights. J. Inequal. Appl. 2020, 119 (2020). https://doi.org/10.1186/s13660-020-02385-x

34. Wang, C., Ye, D.: Some Liouville theorems for Hénon type elliptic equations. J. Funct. Anal. 262, 1705-1727 (2012)

35. Wu, X.: Existence of nontrivial solutions and high energy solutions for Schrödinger-Kirchhoff-type equations in $\mathbb{R}^{N}$. Nonlinear Anal., Real World Appl. 12, 1278-1287 (2011)

36. Xie, Q.L.: Bounded state solution of degenerate Kirchhoff type problem with a critical exponent. J. Math. Anal. Appl. 479, 1-24 (2019)

37. Ye, Y.J.: Blow-up of solutions for a system of higher-order nonlinear Kirchhoff-type equations. Bull. Malays. Math. Sci. Soc. 40, 665-677 (2017)

38. Yu, X.H.: Liouville type theorem for nonlinear elliptic equation involving Grushin operators. Commun. Contemp. Math. 17, 1450050 (2015). https://doi.org/10.1142/S0219199714500503

39. Zhang, C.H., Liu, Z.S.: Multiplicity of nontrivial solutions for a critical degenerate Kirchhoff type problem. Appl. Math. Lett. 69, 87-93 (2017)

40. Zhang, F.B., Du, M.: Existence and asymptotic behavior of positive solutions for Kirchhoff type problems with steep potential well. J. Differ. Equ. 269, 10085-10106 (2020)

\section{Submit your manuscript to a SpringerOpen ${ }^{\mathcal{O}}$ journal and benefit from:}

- Convenient online submission

- Rigorous peer review

- Open access: articles freely available online

- High visibility within the field

Retaining the copyright to your article

Submit your next manuscript at $\boldsymbol{~ s p r i n g e r o p e n . c o m ~}$ 